\title{
Segurança e qualidade higiênico-sanitária em unidades produtoras de refeições comerciais ${ }^{1}$
}

\section{Safety and sanitary quality of food services}

\section{Objetivo}

O objetivo da pesquisa foi analisar as estratégias utilizadas para a gestão da qualidade higiênico-sanitária em unidades produtoras de refeições comerciais de Florianópolis (SC).

\section{Métodos}

A amostra foi formada por 105 unidades produtoras de refeições comerciais. Para a coleta de dados, foi realizada entrevista com os gerentes por meio de um instrumento adaptado que contempla a caracterização dos locais, porte, forma jurídica, capacidade ocupacional, número de refeições servidas e descrição dos sistemas e atividades de gestão de qualidade. Utilizaram-se para as análises o teste qui-quadrado pelo software NTIA 4.2.2 e o teste de $G$ para tabelas de contingência pelo BioEstat 5.0. Para as questões abertas, foram feitas a transcrição dos dados e a análise de informações categorizadas em temas mais frequentes referidos pelos gerentes.

\section{Resultados}

A maioria dos locais (78,8\%) utilizava algum método operacional e/ou de qualidade higiênico-sanitária; 58,9\% utilizavam as boas práticas e $18,6 \%$ o sistema de Análise de Perigos e Pontos Críticos de Controle. Dos locais que não utilizavam nenhuma prática, $51,1 \%$ dos gerentes desconheciam os métodos. Quanto aos investimentos, foi apontada a necessidade de melhorar a estrutura física, os equipamentos e a capacitação dos funcionários.

\footnotetext{
1 Artigo elaborado a partir da dissertação de M.V. EBONE, intitulada "Qualidade higiênico-sanitária em unidades produtoras de refeições comerciais de Florianópolis. Universidade Federal de Santa Catarina; 2010. Apoio: Conselho Nacional de Desenvolvimento Científico e Tecnológico, Programa de Pós-Graduação em Nutrição da Universidade Federal de Santa Catarina e Coordenação de Aperfeiçoamento de Pessoal de Nível Superior.

2 Universidade Federal de Santa Catarina, Programa de Pós-Graduação em Nutrição, Núcleo de Pesquisa de Nutrição em Produção de Refeições. Campus Universitário, Trindade, 88040-900, Florianópolis, SC, Brasil. Correspondência para/ Correspondence to: M.V. EBONE. E-mail: <michele.ebone@gmail.com>.

${ }^{3}$ Universidade Federal de Santa Catarina, Centro de Ciências da Saúde, Departamento de Nutrição. Florianópolis, SC, Brasil.

${ }^{4}$ Universidade Federal de Santa Maria, Departamento de Fitotecnia. Santa Maria, RS, Brasil.
} 
726 | M.V. EBONE et al.

\section{Conclusão}

O estudo monstrou que as unidades produtoras de refeições comerciais necessitam de melhorias para servirem alimentos mais seguros, sendo imprescindível a conscientização dos proprietários e dos gerentes sobre a importância de servir alimentos de qualidade que não causem danos à saúde do comensal.

Termos de indexação: Controle de qualidade. Higiene dos alimentos. Restaurantes.

\section{A B S T R A C T}

\section{Objective}

The objective of this study was to analyze the hygiene and sanitation strategies employed by food services of Florianópolis (SC), Brazil.

\section{Methods}

The sample consisted of 105 food services. Managers were interviewed using an adapted instrument that covers the characterization of the places, their size, their legal form, number of seats, number of meals served and description of the systems and activities related to quality management. The analyses were done by the software NTIA 4.2.2 using the chi-square test and by the software BioEstat 5.0 using the G-test for the contingency tables. The open questions were transcribed and the information was categorized into the most common themes mentioned by the managers for analysis.

\section{Results}

Most establishments (78.8\%) used some quality system or activity, mainly the good practices (58.9\%) and Hazard Analysis and Critical Control Points (HACCP) (18.6\%). More than half $(51.1 \%)$ the managers of establishments that had no quality systems or activities reported not being aware of them. Managers believed it was necessary to improve the facilities, their equipment and utensils, and personnel training.

\section{Conclusion}

Food services need improvements to provide safer foods. Owners and managers have to be made aware of the importance of serving foods that do not jeopardize the health of their customers.

Indexing terms: Quality control. Food hygiene. Restaurants.

\section{N T R O D U ÇÃ O}

As Unidades Produtoras de Refeições (UPR) são essenciais e cada vez mais importantes no sistema alimentar em quase todos os países. Mas, na ausência de um controle rigoroso no processamento, no armazenamento e na distribuição, os alimentos têm potencial para se tornarem fonte importante de doenças. As Doenças Transmitidas por Alimentos (DTA) ${ }^{1}$ são consideradas um problema de saúde pública pela possível abrangência de disseminação devido ao número de refeições realizadas fora de casa atualmente ${ }^{2,3}$.

Assim, as UPR devem seguir diversas regras em função do nível de exposição dos alimentos, variedade de preparações servidas, complexidade das operações efetuadas e armazenamento ${ }^{4}$.
De acordo com a Organização Mundial da Saúde (OMS) ${ }^{5}$, nas UPR, as DTA ligadas à qualidade higiênico-sanitária podem ser ocasionadas por contaminação cruzada, manipuladores, equipamentos e ambiente contaminados, resfriamento e/ou refrigeração e armazenamento inadequados, entre outras causas. Cabe salientar que, no Brasil, entre 1999 e 2008, houve 6062 surtos de DTA, tendo os restaurantes ocupado o segundo lugar das ocorrências, com 19,7\% dos casos ${ }^{6}$.

Para evitar esses surtos e obter alimentos seguros, é imprescindível a adoção de medidas que controlem o alimento desde sua origem até o consumo. Assim, a manipulação dos alimentos a partir das Boas Práticas (BP) e o uso do Sistema de Análise de Perigos e Pontos Críticos de Controle (APPCC) são fundamentais para a redução dos perigos de origem alimentar ${ }^{4,7}$. 
Assim, o objetivo desse estudo foi caracterizar as UPR em bufê por peso, fast-food e churrascaria, com o intuito de analisar as estratégias utilizadas para a gestão da qualidade higiênico-sanitária.

\section{MÉ T O D OS}

Desenvolvido em Florianópolis (SC), o estudo adotou os procedimentos previstos para a pesquisa de natureza descritiva ${ }^{8,9}$. A determinação da amostra foi baseada nas listas das Páginas Amarelas online, Listel e site Hagah. Quanto à representatividade numérica, para os tipos fast-food e churrascarias, utilizaram-se como amostra $100 \%$ dos estabelecimentos localizados por meio de contato telefônico e que aceitaram participar da entrevista.

A amostra de bufês por peso contemplou o percentual de $50 \%$ da população dessa classificação, apresentando um número maior de estabelecimentos, sendo considerada uma proporção representativa. Assim, as comparações entre as UPR ficaram mais adequadas a partir da utilização de um número semelhante de locais. A escolha dos bufês por peso foi aleatória, de acordo com a amostra previamente definida; esses estabelecimentos foram localizados por meio de contato telefônico e foram selecionados os que aceitaram participar da entrevista.

Por fim, considerou-se o tamanho total da amostra (n) igual a 105 UPR comerciais: 48 bufês por peso, 16 churrascarias e 41 fast-food.

Para a coleta de dados, entrevistaram-se os gerentes dos locais e utilizaram-se as questões sobre sistemas de qualidade do instrumento de pesquisa de Cavalli $^{10}$, aplicadas entre novembro de 2006 e julho de 2007 por entrevistadores capacitados.

O questionário continha questões sobre o porte da empresa (micro, pequena, média ou grande), o número de refeições servidas (movimentação do público) e a descrição dos sistemas e/ou atividades de gestão de qualidade: Qualidade Total (TQM ou TQC), Série ISO 9000, Programa $5 \mathrm{~S}$, Sistema APPCC, BP, check list. O questionário contemplava também as seguintes questões abertas: "o que é servir alimento seguro", "quais investimentos necessários para melhorar a qualidade do alimento servido" e "o que a empresa espera de apoio governamental para o setor". Questionou-se, ainda, a razão da não implantação das BP e/ou sistema APPCC.

O tratamento estatístico utilizado foi a análise de associação pelo teste qui-quadrado, em nível de $5 \%$ de significância. Utilizou-se para a análise estatística o software NTIA versão 4.2.2 (http://www.embrapa.br/english/portfolio/ information\%20science/), produzido pela Empresa Brasileira de Pesquisa Agropecuária (EMBRAPA) de Campinas (SP). Para os casos que não atenderam às pressuposições do qui-quadrado, foi utilizado o Teste de G em nível de 5\% de significância para tabelas de contingência no BioEstat 5.0.

Para as questões abertas, utilizou-se a análise de informações por meio da transcrição dos dados obtidos dos questionários, que foram posteriormente categorizados em temas mais frequentes referidos pelos gerentes ${ }^{9}$.

A pesquisa foi submetida ao Comitê de Ética em Pesquisa com Seres Humanos da Universidade Federal de Santa Catarina. O comitê emitiu parecer da não necessidade de submissão ao Comitê de Ética em Pesquisa com Seres Humanos do projeto de estudo, considerando as questões formuladas para as empresas.

\section{RESULTADOSE DISCUSSÃO}

Em relação ao tipo da UPR, $46 \%$ eram bufês por peso, 39\% fast-food e 15\% churrascarias. Quanto ao porte, $95 \%$ enquadravam-se em micro e pequenas empresas, $3 \%$ em médias e $2 \%$ em grandes empresas.

Observou-se que $78,85 \%$ das UPR adotavam métodos de controle de qualidade operacional e/ou higiênico-sanitário e que não houve relação significativa entre a existência de atividades e/ou sistemas de qualidade, o tipo (Teste-G: 2,99; $\alpha$ : $5 \%$ ) e o porte da UPR (Teste-G: 1,$90 ; \alpha$ : $5 \%)$. 
Estudo realizado em UPR na Turquia sobre as dificuldades e as barreiras enfrentadas na aplicação de sistemas para a segurança alimentar apontou que apenas 33\% delas tinham sistemas, mesmo com a legislação do país estando de acordo com a União Europeia"11.

Na presente pesquisa, foi evidenciada a predominância do uso de um tipo de método $(62,6 \%)$. Pode-se destacar a baixa adesão às BP (58,9\%), que apareceram como sistema único e/ou associadas a outras atividades, como check list, Programa Alimentos Seguros, Sistema 5S, sistema próprio, cursos de manipulação, recebimento dos alimentos em temperatura e local adequados, acompanhamento de profissional da área de alimentos, análise microbiológica da água, dos alimentos, dos utensílios e funcionários, cardápio padrão, Qualidade de Serviço e Limpeza (QSE), Comunicação, Cooperação e Coordenação (3C), ISO 9000 e qualidade total (Tabela 1).

Para alguns autores, as atividades citadas (Tabela 1) formam um conjunto de métodos que se complementam: para a qualidade dos alimentos, por exemplo, usam-se as normas ISO e qualidade total; já para a segurança alimentar, as BP e o sistema APPCC ${ }^{12,13}$.

Observou-se uma relação não significativa (Teste-G: 16,$54 ; \alpha$ : 5\%) entre o número de refeições e o uso de sistemas e/ou atividades de qualidade. Em estudo semelhante realizado em UPR de outras regiões do Brasil, foi encontrada uma relação significativa entre o número de refeições servidas e o uso de sistemas e/ou atividades de qualidade ${ }^{14}$. Contudo, cabe destacar que os sistemas de qualidade higiênico-sanitária, como as BP e o sistema APPCC, são obrigatórios para as empresas do setor, independentemente do tipo, do porte ou do número de refeições servidas ${ }^{15,16}$, sendo essencial a fiscalização pela vigilância sanitária.

Observou-se também uma relação não significativa (Teste-G: 3,4510; $\alpha$ : $5 \%$ ) entre o porte das empresas e o uso de sistemas/atividades de qualidade.

De acordo com o Guia Alimentar para a População Brasileira ${ }^{7}$, para atingir a qualidade sanitária dos alimentos, devem ser adotadas medidas preventivas e de controle desde a origem até o consumo do alimento, sendo a manipulação adequada essencial para redução dos riscos de DTA.

Nos locais investigados, o sistema APPCC era implantado quando utilizado mais de um método para o controle de qualidade, evidenciando-se sua aplicação em conjunto com as BP $(18,6 \%)$ (Tabela 1). Pode-se destacar que, mesmo não havendo adesão por todos os locais, sua presença em conjunto com as BP é considerada positiva, uma vez que, antes da implantação do APPCC, é necessário a empresa preencher os pré-requisitos, como as $\mathrm{BP}^{17-19}$, tornando a aplicação do sistema constante e com menos problemas ${ }^{20-22}$.

Tabela 1. Percentual das atividades e/ou sistemas de qualidade adotados pelas unidades produtoras de refeições comerciais. Florianópolis (SC), 2007

\begin{tabular}{lclllr}
\hline \multicolumn{5}{c}{ Tipos de atividades e sistemas de qualidade utilizados } \\
\hline Somente um & $\%$ & Dois & $\%$ & Mais de dois & $\%$ \\
\hline BP & 28,9 & APPCC e BP & 4,8 & APPCC/BP/outros $^{* 3}$ & 13,2 \\
Sistema próprio & 18,1 & BP e Check list & 3,6 & BP/Check list/outros $^{* 4}$ & 8,4 \\
Check list $^{*}$ & 12,0 & Outros $^{* 2}$ & 4,8 & Check list/reuniões mensais/listagem de rotinas $^{*}$ & 1,2 \\
Outros $^{* 1}$ & 3,6 & & & 5S/Check list/acompanhamento da produção & 1,2 \\
\hline Total & 62,6 & & 13,2 & & 24,0 \\
\hline
\end{tabular}

Outros: soma do percentual de métodos citados com frequência menor ou igual a 2,5\%; Outros ${ }^{* 1}$ : Acompanhamento da produção e Produtos de qualidade; Outros ${ }^{\star 2}$ : 5 S e PAS, APPCC e PAS, Check list e rotação de atividades e Acompanhamento da produção/controle dos funcionários; Outros $^{* 3}$ : Check list, PAS, 5S, sistema próprio, cursos de manipulação, recebimento dos alimentos em temperatura e local adequados, acompanhamento de profissional da área, qualidade total; Outros*4: análise microbiológica da água, dos alimentos, dos utensílios e funcionários, cardápio padrão, cursos de manipulação, QSE (qualidade de serviço e limpeza), 3C (comunicação, cooperação e coordenação), ISO 9000, Qualidade total, 5S, PAS; Total de serviços de alimentação que adotam os sistemas: 83. 
A adesão ao sistema APPCC pode ser considerada baixa, comparando-a com uma pesquisa realizada no Reino Unido, que indicou que $60 \%$ dos serviços de alimentação de porte pequeno e médio implementavam esse sistema ${ }^{23}$.

Deve-se salientar sua obrigatoriedade, visto que o sistema APPCC pode ser usado para controlar o comportamento dos funcionários nas operações que são essenciais para a segurança dos alimentos ${ }^{24}$, ajudando a identificação dos perigos, reduzindo os riscos de DTA ${ }^{25}$, tornando as inspeções mais fáceis e completas, aumentando o cumprimento da legislação 22 e a confiança dos consumidores e diminuindo os custos ${ }^{20}$.

Entretanto, as UPR possuem dificuldades de implementar o sistema APPCC em seu formato original ${ }^{26}$, e o governo e o setor produtivo reconhecem que pode haver obstáculos na aplicação desse sistema, principalmente nas empresas de pequeno porte ${ }^{27}$.

\section{Razões citadas para a não adoção das boas práticas e do sistema APPCC}

Em relação às razões apontadas pelos gerentes para a não adoção das BP e do sistema APPCC (Tabela 2), pode-se destacar que 51,1\% dos gerentes desconheciam as ferramentas. Verificou-se, ainda, não haver relação significativa entre os tipos de UPR e as respostas $\left(\chi^{2}=23,81\right.$; $\alpha=5,0 \%)$, e entre o porte e as respostas $\left(\chi^{2}=14,06\right.$; $\alpha=5,0 \%$ ).
Estudo realizado no Brasil por Cavalli \& Salay ${ }^{14}$ identificou que as razões para os gerentes não utilizarem o sistema APPCC e as BP eram: desconhecimento dos sistemas (22\%), o fato de o controle ser feito pelo próprio gerente e/ou funcionários (14\%), o fato de a empresa ser considerada pequena $(12 \%)$ e os elevados custos de implantação (10\%).

$\mathrm{Na}$ Irlanda, 78,0\% dos gerentes de um estudo feito em UPR declararam desconhecer as exigências da legislação para o setor ${ }^{3}$. Já na Turquia, pouco menos da metade $(43,0 \%)$ dos gerentes desconhecia o sistema APPCC, e 92,2\% concordaram que havia a necessidade de aumentar a fiscalização ${ }^{11}$.

Na presente pesquisa, $17,8 \%$ dos gerentes alegaram possuir método próprio para o controle da qualidade (Tabela 2), como provar gosto dos alimentos. Contudo, esse método é considerado insuficiente por alguns autores ${ }^{5,28}$ e incompatível com a legislação sanitária brasileira ${ }^{15,16}$.

A falta de recursos financeiros é uma limitação também assinalada em outros estudos ${ }^{19,22}$. No entanto, os gerentes devem considerar benefícios como economia em longo prazo; redução dos processos contra os locais; menor desperdício devido ao melhor transporte, armazenagem e processamento; maior empenho dos funcionários ${ }^{24}$; maior confiança dos clientes; cumprimento da legislação e melhor administração ${ }^{11}$.

Em relação à resposta "falta de cobrança pelo cliente" (Tabela 2), a World Health

Tabela 2. Percentual das razões citadas pelos gerentes para a não-adoção das boas práticas e do sistema APPCC nas unidades produtoras de refeições comerciais. Florianópolis (SC), $2007^{1}$.

\begin{tabular}{|c|c|}
\hline Respostas & $\%$ \\
\hline Desconhece os métodos & 51,1 \\
\hline A empresa dispõe de método próprio & 17,8 \\
\hline Fatores econômicos/financeiros & 11,1 \\
\hline Não há cobrança pela vigilância sanitária & 4,4 \\
\hline Não há cobrança pela vigilância sanitária e não há cobrança pelo cliente & 4,4 \\
\hline Desconhece o(s) método(s), e a empresa dispõe de método próprio ou não há cobrança pela vigilância sanitária & 4,4 \\
\hline Outros $^{2}$ & 6,7 \\
\hline Total & 100,0 \\
\hline
\end{tabular}

${ }^{1}$ Responderam a esta questão os 45 serviços de alimentação que não adotavam sistemas de qualidade operacional e/ou sanitário; ${ }^{2}$ Outros: A empresa é pequena; a empresa é nova, está implantando; método próprio e acha que não é necessário. Soma do percentual de métodos citados com frequência menor ou igual a $2,5 \%$. 
Organization ${ }^{20}$ confirma que a exigência do consumidor é importante, pois estimula os gerentes dos locais a melhorarem seus serviços com a implantação do sistema APPCC.

Quanto à resposta dos gerentes de que as BP e o sistema APPCC não eram necessários (Tabela 2), foram encontrados dados semelhantes em estudo realizado em UPR da Espanha. Contudo, os autores espanhóis reforçaram a importância dos sistemas, concluindo, por meio de testes microbiológicos, que o treinamento dos manipuladores em BP e APPCC contribuiu para que fossem servidos alimentos mais seguros ${ }^{13}$.

Pode-se considerar de suma importância a valorização dos sistemas pelos gerentes do setor, pois estudo feito na Inglaterra identificou que os gerentes de UPR acreditam que suas atitudes em relação aos sistemas afetam o comportamento dos funcionários ${ }^{22}$. Desse modo, se o gerente não acredita ser necessário o APPCC, o funcionário também agirá assim, sendo imprescindível rever essa postura².

\section{Significados de servir alimento seguro para os gerentes}

Os relatos dos gerentes sobre o significado de servir alimento seguro foram categorizados em temas e indicaram o conhecimento de uma variedade de ações a serem desenvolvidas (Quadro 1).

Houve, nas respostas dos gerentes (Quadro 1), coerência nos temas referidos: de acordo com a $\mathrm{OMS}^{29}$, alimento seguro significa que a água e os alimentos servidos não estarão contaminados com microrganismos ou elementos químicos tóxicos em quantidades que possam causar DTA.

Pode-se afirmar que alguns gerentes têm clareza do que deve ser controlado durante a produção das refeições quando comparadas as respostas (Quadro 1) com as diretrizes preconizadas no manual "Cinco chaves para uma alimentação mais segura" 29 da OMS, que traz a importância de manter a limpeza, de separar os alimen-

Quadro 1. Temas recorrentes referidos pelos gerentes das unidades produtoras de refeições comerciais sobre o significado de servir alimento seguro. Florianópolis (SC), 2007.

\begin{tabular}{|c|c|}
\hline Categorias & Descrição dos temas \\
\hline Qualidade & $\begin{array}{l}\text { Utilizar produtos de qualidade adquiridos de bons fornecedores, respeitando o prazo de valida- } \\
\text { de, armazenados adequadamente e manipulados, seguindo as normas de higiene e da vigilân- } \\
\text { cia sanitária. }\end{array}$ \\
\hline Higiene & $\begin{array}{l}\text { Usar uniformes adequados e luvas. } \\
\text { Manter a higiene das áreas em geral e em todas as etapas de produção, com correta limpeza e } \\
\text { higienização da área física, das mãos dos manipuladores e dos alimentos. } \\
\text { Garantir ao cliente que os alimentos não vão prejudicá-lo quando consumidos, servindo ali- } \\
\text { mentos livres de contaminações, fazendo controle de pragas. }\end{array}$ \\
\hline Sistemas de qualidade & $\begin{array}{l}\text { Ter as boas práticas e o Programa de Alimentos Seguros. } \\
\text { Fazer o controle da temperatura das preparações. }\end{array}$ \\
\hline Gestão do fluxo produtivo & $\begin{array}{l}\text { Conhecer a origem da matéria-prima, com a escolha adequada do fornecedor; cuidados com a } \\
\text { recepção, tempo de preparo, manipulação, manutenção pós-preparo e distribuição. }\end{array}$ \\
\hline Produção supervisionada & $\begin{array}{l}\text { Ter a produção supervisionada por nutricionista que oriente os manipuladores sobre higiene e } \\
\text { armazenamento dos alimentos. }\end{array}$ \\
\hline Recursos humanos & $\begin{array}{l}\text { Poder dispor de funcionários responsáveis, em bom estado de saúde e com exames médicos em } \\
\text { dia, que seguem as regras de higiene pessoal, capacitados e treinados para seguirem as regras } \\
\text { de segurança na manipulação dos alimentos. }\end{array}$ \\
\hline Satisfação do cliente/marketing & $\begin{array}{l}\text { Preparar os alimentos no dia do seu consumo com amor, carinho, respeito e compromisso, sem } \\
\text { pressa; servindo uma alimentação de qualidade, saborosa e com boa aparência, com alimentos } \\
\text { variados, orgânicos, frescos, da época; comida caseira; preparações vegetarianas, que saciem e } \\
\text { proporcionem bem-estar. Valorização do prato principal, não havendo reaproveitamento, mas } \\
\text { apenas armazenagem de amostras; tendo bom atendimento para satisfação, fidelização e re- } \\
\text { torno do cliente. }\end{array}$ \\
\hline
\end{tabular}


tos crus dos cozidos, de cozinhar bem os alimentos, de mantê-los em temperaturas seguras e de usar água potável e matérias-primas confiáveis.

Já sobre os sistemas, os gerentes citaram as $\mathrm{BP}$, mas não o sistema APPCC, diferindo dos resultados encontrados em estudo realizado em UPR na Turquia, no qual os gerentes acreditam que o sistema APPCC melhora a segurança dos alimentos ${ }^{11}$.

\section{Investimentos em controle de qualidade}

Quando questionados sobre a necessidade de investimentos em controle de qualidade e segurança dos alimentos, verificaram-se diversas respostas, que foram separadas pelos tipos dos locais e tipos de investimento (Quadro 2).

Pode-se observar que os gerentes concordaram haver necessidade de investimentos em recursos humanos, em capacitação e nas atividades e/ou sistemas para a segurança do alimento (Quadro 2). A concretização dessas ações é impor- tante para o setor, pois as BP, o sistema APPCC e a capacitação dos manipuladores são preconizados pela legislação brasileira ${ }^{15,16}$, devendo os gerentes e os proprietários das UPR investir em medidas preventivas e de controle da produção $0^{7,20}$.

Quanto à resposta "Ter um bom relacionamento com os funcionários" (Quadro 2), de acordo com Ko ${ }^{30}$, um ambiente positivo de trabalho e o reconhecimento individual são fatores motivacionais que contribuem para a lealdade, para uma melhor atitude e para a produtividade. Frequentemente, os funcionários estão desmotivados, com atitudes negativas no que diz respeito à segurança dos alimentos ${ }^{19}$.

Os equipamentos, que são essenciais para a realização do trabalho e que podem causar impacto na segurança dos alimentos ${ }^{26}$, também foram apontados pelos gerentes dos bufês e dos fast-food como carentes de melhoria (Quadro 2) e estão entre os primeiros fatores a serem considerados antes da implementação do sistema APPC ${ }^{19}$, em conjunto com o tamanho da co-

Quadro 2. Necessidades de investimentos citados pelos gerentes das unidades produtoras de refeições comerciais. Florianópolis (SC), 2007.

\begin{tabular}{|c|c|c|c|}
\hline Tipologias/tipos de investimentos & Bufês por peso & Churrascarias & Fast-food \\
\hline Estrutural & $\begin{array}{l}\text { - Reformar a estrutura física. } \\
\text { - Comprar equipamentos (como } \\
\text { câmara fria, Pass through, for- } \\
\text { no combinado, boiler, máqui- } \\
\text { na de lavar louça, ar-condicio- } \\
\text { nado). } \\
\text { - Comprar utensílios. }\end{array}$ & & $\begin{array}{l}\text { - Investir na área física. } \\
\text { - Investir em equipamentos. }\end{array}$ \\
\hline Recursos humanos & $\begin{array}{l}\text { - Investir em treinamento sobre } \\
\text { os controles de qualidade. } \\
\text { - Contratar um nutricionista. } \\
\text { - Ter um bom relacionamento } \\
\text { com os funcionários. }\end{array}$ & $\begin{array}{l}\text { - Investir em cursos. } \\
\text { - Investir em um profissional } \\
\text { qualificado para realizar os } \\
\text { treinamentos. } \\
\text { - Contratar profissionais ca- } \\
\text { pacitados. } \\
\text { - Selecionar e treinar melhor } \\
\text { os funcionários. }\end{array}$ & $\begin{array}{l}\text { - Ter supervisão constante de um } \\
\text { responsável técnico/nutricionis- } \\
\text { ta. } \\
\text { - Proporcionar cursos de manipu- } \\
\text { lação e capacitação dos funcio- } \\
\text { nários, de } 6 \text { em } 6 \text { meses. } \\
\text { - Contratar profissionais mais qua- } \\
\text { lificados, com cursos profissiona- } \\
\text { lizantes. }\end{array}$ \\
\hline Processos & $\begin{array}{l}\text { - Investir em boas práticas. } \\
\text { - Controlar os fornecedores. } \\
\text { - Acompanhar a produção do ali- } \\
\text { mento desde a sua origem. }\end{array}$ & $\begin{array}{l}\text { - Implantar sistemas de con- } \\
\text { trole de qualidade. }\end{array}$ & $\begin{array}{l}\text { - Investir em sistemas para segu- } \\
\text { rança dos alimentos, como o Pro- } \\
\text { grama de Alimentos Seguros. } \\
\text { - Comprar produtos adequados } \\
\text { para a higienização. }\end{array}$ \\
\hline Geral & & - Investir mais na empresa. & \\
\hline
\end{tabular}


zinha ${ }^{31}$, o leiaute e o fluxo da produção ${ }^{32}$. Portanto, a área deve ser projetada com os equipamentos apropriados para facilitar a higienização e para evitar a contaminação cruzada ${ }^{20}$.

\section{Expectativas de apoio governamental}

No que se refere às expectativas dos gerentes de apoio governamental para a qualidade e segurança dos alimentos, a resposta mais frequente foi que eles não esperavam nenhum apoio (49\%) (Tabela 3).

Pode-se destacar que o apoio do governo é muito importante para a implementação de programas para a segurança dos alimentos, devendo as metas para o setor das autoridades reguladoras ser claras, uniformes e amplamente divulgadas ${ }^{19}$. Na Turquia, os gerentes das empresas de alimentação também acreditam que o governo deveria dar mais apoio ao setor para a implementação de sistemas ${ }^{11}$.

Quanto à resposta sobre a necessidade de melhorias nas leis e no sistema de fiscalização com auxílio da vigilância sanitária (Tabela 3), a World Health Organization ${ }^{33}$ destaca que o governo precisa ter leis e padrões atualizados para a inspeção, que deve ser feita por pessoas honestas, eficientes, treinadas e qualificadas. A inspeção é essencial, pois é parte integrante dos sistemas para controle dos alimentos ${ }^{34}$. Contudo, além da fiscalização, é fundamental que se estabeleçam parcerias com setores de apoio ao setor de alimentos com objetivo de dar suporte à imple- mentação da legislação por meio de capacitações, orientações técnicas e assessorias aos estabelecimentos ${ }^{7}$.

As agências reguladoras, indústrias, instituições educacionais e consultores devem ser encorajados pelo governo para assistirem as pequenas empresas ${ }^{24}$, pois um papel importante dos sistemas de controle de alimentos é a divulgação de informações e a educação dos clientes, colaboradores, proprietários e fornecedores, atingindo, assim, toda a cadeia produtiva, do campo à mesa ${ }^{33}$.

\section{O N CLUS Ã O}

A pesquisa monstrou que, embora a maioria (78,8\%) das UPR pesquisadas trabalhasse com atividades e sistemas de qualidade operacional e/ou higiênico-sanitária, elas ainda necessitavam de melhorias, pois apesar de os gerentes saberem o que é necessário para ofertar alimentos seguros, apenas 58,9\% dos locais tinham as BP, e 18,6\% o sistema APPCC. Isso pode ser explicado pelo desconhecimento desses sistemas, alegado por mais da metade dos gerentes $(51,1 \%)$. Esses dados e os relatos sobre os investimentos necessários demonstraram que deve haver um maior envolvimento por parte dos gerentes e dos proprietários para uma adequação às exigências regulatórias para o setor.

Ainda que os gerentes não esperem apoio do governo, é essencial a colaboração dos órgãos competentes responsáveis pelo setor para uma

Tabela 3. Expectativas dos gerentes das unidades produtoras de refeições comerciais quanto ao apoio governamental para contribuir com a qualidade e segurança dos alimentos. Florianópolis (SC), 2007.

\begin{tabular}{lcc}
\hline Respostas & $\mathrm{n}^{1}$ & $\%$ \\
\hline - Não esperam nada. & 50 & 49,0 \\
- Cursos de manipulação, profissionalizantes e programas de capacitação gratuitos. & 18 & 17,6 \\
- Redução de impostos e encargos trabalhistas. & 17 & 16,6 \\
- Melhoria das leis e do sistema de fiscalização com auxílio da vigilância sanitária. & 6 & 12,7 \\
- Melhor fiscalização das matérias-primas, com incentivo para agricultura e pecuária. & 6 & 5,8 \\
- Apoio financeiro. & 3 & 2,8 \\
- Campanhas para o setor e apoio de instituições, como o Programa de Alimentos Seguros. & 2 & 1,9 \\
- Apoio geral, permanente; não soube informar. & & \\
\hline
\end{tabular}

$\mathrm{n}^{1}$ : Cada gerente declarou mais de uma expectativa. 
correta fiscalização e desenvolvimento de programas de conscientização dos gerentes e dos manipuladores quanto à importância e à obrigatoriedade da implementação dos sistemas para a gestão da qualidade higiênico-sanitária.

Por fim, sugere-se que esta pesquisa com os gerentes/proprietários seja realizada também com os funcionários e em diferentes tipos de UPR, como hotéis, restaurantes à la carte e ambulantes, para melhor compreensão das especificidades na área de produção de refeições.

\section{A GRADECIMENTOS}

A participação dos gerentes das unidades produtoras de refeições, ao Conselho Nacional de Desenvolvimento Científico e Tecnológico, pelo financiamento feito por meio do Edital MCT-CNPq/MS-DAB/SAS n 51/2005, sob o número de processo 402094/2005-0, ao Programa de Pós-Graduação em Nutrição da Universidade Federal de Santa Catarina e ao Programa de Fomento à Pós-Graduação da Coordenação de Aperfeiçoamento de Pessoal de Nível Superior.

\section{COLABORADORES}

M.V. EBONE participou da concepção e estrutura dos dados, da coleta, da análise, da interpretação dos dados e da elaboração do artigo. S.B. CAVALLI participou da concepção e estrutura dos dados e da revisão crítica do artigo. S.J. LOPES participou da definição da amostra, da análise estatística dos dados e da revisão crítica do artigo.

\section{REFER Ê N CIAS}

1. Organização Mundial de Saúde. Implementation of initiatives to manage food safety. 2000 [cited 2009 May 19]. Available from: <http://www. searo.who.int/LinkFiles/RC_53_rc53-6_r1_con. $\mathrm{pdf}>$.

2. Motarjemi Y, Kaferstein F. Food safety, hazard analysis and critical control point and the increase in foodborne diseases: a paradox? Food Control. 1999; 10(4-5):325-33. doi: 10.1016/S0956-7135 (99)00008-0.
3. Bolton DJ, Meally A, Blair IS, McDowell DA, Cowan C. Food safety knowledge of head chefs and catering managers in Ireland. Food Control. 2008; 19(3):291-300. doi: 10.1016/j.foodcont.2007.0 4.006.

4. Organização Mundial de Saúde. Exposure assessment of microbiological hazards in food guidelines. 2008. Microbiological risk assessment series [cited 2009 Jan 21]. Available from: <http:// www.codexalimentarius.net/search/advanced search.do>.

5. Organização Mundial de Saúde. Foodborne disease outbreaks: guidelines for investigation and control. 2009 [cited 2009 May 21]. Available from: <http:// www.who.int/foodsafety/publications/foodborne_ disease/fdbmanual/en/index.html>.

6. Brasil. Ministério da Saúde. Secretaria de Vigilância em Saúde. Análise epidemiológica dos surtos de doenças transmitidas por alimentos no Brasil. Brasília: MS; 2008 [acesso 2009 dez 3]. Disponível em: <http://portal.saude.gov.br/portal/arquivos/ pdf/surtos_dta_15.pdf94>.

7. Brasil. Ministério da Saúde. Secretaria de Atenção à Saúde. Guia alimentar para a população brasileira: promovendo a alimentação saudável [Edição Especial]. Brasília: MS; 2005. Série A. Normas e Manuais Técnicos.

8. Contandriopoulos AP, Champagne F, Potvin L, Denis JL, Boyle P. Saber preparar uma pesquisa: definição estrutura financiamento. São Paulo: Hucitec; 1997.

9. Richardson RJ. Pesquisa social: métodos e técnicas. São Paulo: Atlas; 1999.

10. Cavalli SB. Sistemas de controle de qualidade e segurança do alimento, processo produtivo e recursos humanos em unidades de alimentação comercial de Campinas (SP) e Porto Alegre (RS) [tese]. Campinas: Unicamp; 2010.

11. Bas M, Yüksel M, Çavuooflu T. Difficulties and barriers for the implementing of HACCP and food safety systems in food businesses in Turkey. Food Control. 2007; 18(2):124-130. doi: 10.1016/j.food cont.2005.09.002.

12. Raspor P. Total food chain safety: how good practices can contribute? Trends Food Sci Technol. 2008; 19: 405-12. doi: 10.1016/j.tifs.2007.08.009.

13. Soriano JM, Font G, Moltó JC, Mañes J. Enterotoxigenic staphylococci and their toxins in restaurant foods. Trends Food Sci Technol. 2002; 13(2):60-7. doi: 10.1016/S0924-2244(02)00030-4.

14. Cavalli SB, Salay E. Food quality and safety control activities in commercial foodservices in the cities of Campinas (SP) and Porto Alegre (RS), Brazil. 
Wiley. 2004. [cited 2010 Jan 18]. Available from: <http://www3.interscience.wiley.com/journal/ 118816638/abstract? CRETRY $=1 \&$ SRETRY $=0>$.

15. Brasil. Ministério da Saúde. Portaria nº 1428 de 26 de novembro de 1993. Regulamento Técnico para Inspeção Sanitária de Alimentos. Diretrizes para o estabelecimento de Boas Práticas de Produção e Prestação de Serviços na área de Alimentos. Regulamento Técnico para o Estabelecimento de Padrão de Identidade e Qualidade (PIQ's) para serviços e produtos na área de Alimentos. Brasília: MS; 1993 [acesso 2010 ago 30]. Disponível em: <http://www. anvisa.gov.br/legis/portarias/1428_93.htm>.

16. Brasil. Ministério da Saúde. Resolução RDC n²16, de 15 de setembro de 2004. Dispõe sobre Regulamento Técnico de Boas Práticas para Serviços de Alimentação. Brasília: MS; 2004 [acesso 2010 ago 30]. Disponível em: <http://e-legis.anvisa.gov.br/ leisref/public/showAct.php?id=12546>.

17. Sperber WH. Auditing and verification of food safety and HACCP. Food Control. 1998; 9(2-3): 157-62. doi: 10.1016/S0956-7135(97)00068-6.

18. Jeng H-YJ, Fang TJ. Food safety control system in Taiwan: the example of food service sector. Food Control. 2003; 14(5):317-22. doi: 10.1016/S0956-7 135(02)00096-8.

19. Bas M, Ersun AS, Kivanç G. Implementation of HACCP and prerequisite programs in food businesses in Turkey. Food Control. 2006; 17(2):118-26. doi: 10.1016/j.foodcont.2004.09.0 10.

20. World Health Organization. Strategies for Implementing HACCP in Small and/or Less Developed Businesses. Food Safety Programme WHO/SDE/PHE/FOS/99.7. 1999 [cited 2009 Jan 21]. Available from: <http://www.who.int/fsf>.

21. Mortimore S. How to make HACCP really worn in practice. Food Control. 2001; 12(4):209-15. doi: 10.1016/S0956-7135(01)00017-2.

22. Eves A, Dervisi P. Experiences of the implementation and operation of hazard analysis critical control points in the food service sector. Int J Hosp Manag. 2005; 24(1):3-19. doi: 10.1016/j.jhm.2004.04.0 05.

23. Walker E, Pritchard C, Forsythe S. Hazard analysis critical control point and prerequisite programme implementation in small and medium size food business. Food Control. 2003; 14(3):169-174. doi: 10.1016/S0956-7135(02)00061-0.

24. World Health Organization. The role of health workers in food safety. 1999 [cited 2009 May 19].
Available from: <www.who.int/entity/foodsafety/ publications/capacity/ en/toc.pdf>.

25. American Dietetic Association. Position of the American Dietetic Association: food and water safety. J Am Dietetic Assoc. 2009; 109(8):1449-60.

26. Taylor E. A new method of HACCP for the catering and food service industry. Food Control. 2008; 19: 126-34. doi: 10.1016/j.foodcont.2007.02.013.

27. Codex Alimentarius Commission. Recommended international code of practice general principles of food hygiene. 2003 [cited 2008 May 15]. Available from: <http://www.codexalimentarius.net/web/ standard_list.jsp>.

28. Walker E, Pritchard C, Forsythe S. Food handlers' hygiene knowledge in small food businesses. Food Control. 2003; 14(5):339-43. doi: 10.1016/\$0956-7 135(02)00101-9.

29. Organização Mundial de Saúde. Departamento de Segurança Alimentar. Zoonoses e Doenças de Origem Alimentar. Cinco chaves para uma alimentação mais segura: manual. Geneva: WHO; 2006 [acesso 2008 maio 15]. Disponível em: <http:// www.who. int/foodsafety/consumer/5keysmanual/ en/index.html>.

30. Ko W-H. Evaluating food safety perceptions and practices for agricultural food handler. Food Control. 2010; 21(4):450-55 doi: 10.1016/j.food cont.2009.07.005.

31. Soriano JM, Rico H, Moltó JC, Mañes J. Effect of introductioon of HACCP on the microbiological quality of some restaurant meals. Food Control. 2002; 13(4):253-61. doi: 10.1016/S0956-7135(02) 00023-3.

32. Sun Y-M, Ockerman HW. A review of the needs and current applications of hazard analysis and critical control point (HACCP) system in foodservice areas. Food Control. 2005; 16(4):325-32. doi: 10.10 16/j.foodcont.2004.03.012.

33. World Health Organization. Assuring food safety and quality: guidelines for strengthening national food control systems. 2009 [cited 2009 Nov 19]. Available from: <http://www.who.int/foodsafety/ publications/capacity/en/Englsih_Guidelines_ Food_control.pdf>.

34. World Health Organization. WHO global strategy for food safety: safer food for better health. Geneva: WHO; 2002.

Recebido em: 5/1/2011

Versão final reapresentada em: 1/4/2011 Aprovado em: 13/5/2011 